\title{
A New Collocation-Type Method for Hammerstein Integral Equations
}

\author{
By Sunil Kumar and Ian H. Sloan
}

Abstract. We consider Hammerstcin equations of the form

$$
y(t)=f(t)+\int_{a}^{b} k(t, s) g(s, y(s)) d s, \quad t \in[a, b],
$$

and present a new method for solving them numerically. The method is a collocation method applied not to the equation in its original form, but rather to an equivalent equation for $z(t):=g(t, y(t))$. The desired approximation to $y$ is then obtained by use of the (exact) equation

$$
y(t)=f(t)+\int_{a}^{\dot{b}} k(t, s) z(s) d s, \quad t \in[a, b] .
$$

Advantages of this method, compared with the direct collocation approximation for $y$, are discussed. The main result in the paper is that, under suitable conditions, the resulting approximation to $y$ converges to the exact solution at a rate at least equal to that of the best approximation to $z$ from the space in which the collocation solution is sought.

1. Introduction. We present a new collocation-type method for the numerical solution of nonlinear integral equations of Hammerstein type. These equations take the form

$$
y(t)=f(t)+\int_{a}^{b} k(t, s) g(s, y(s)) d s, \quad t \in[a, b],
$$

where $-\infty<a<b<\infty, f, k$, and $g$ are known functions and $y$ is the solution to be determined. The function $g(s, v)$ is assumed to be nonlinear in $v$. Appropriate smoothness assumptions on $f, k$, and $g$, to be made later, will ensure that, in a suitable Banach space, the right-hand side of Eq. (1.1) defines a completely continuous operator acting on $y$.

The standard collocation method [2, p. 700] approximates the solution $y$ of Eq. (1.1) by $w_{n}$, where

$$
w_{n}(t)=\sum_{j=1}^{n} b_{n j} u_{n j}(t), \quad t \in[a, b],
$$

with the functions $u_{n 1}, \ldots, u_{n n}$ being known, and the unknown coefficients $b_{n 1}, \ldots, b_{n n}$ being determined by requiring $w_{n}$ to satisfy the $n$ nonlinear equations

$$
w_{n}\left(\tau_{n i}\right)=f\left(\tau_{n i}\right)+\int_{a}^{b} k\left(\tau_{n i}, s\right) g\left(s, w_{n}(s)\right) d s, \quad i=1, \ldots, n,
$$

Received February 11, 1986; revised June 17, 1986.

1980 Mathematics Subject Classification. Primary 65R20, 45G10. 
for $n$ distinct (collocation) points $\tau_{n 1}, \ldots, \tau_{n n}$ in $[a, b]$. The convergence of $w_{n}$ to $y$ may be proved, under suitable conditions on $u_{n 1}, \ldots, u_{n n}$ and $\tau_{n 1}, \ldots, \tau_{n n}$, by applying the general theory of [19].

Substitution of (1.2) into (1.3) leads to a system of nonlinear equations for $b_{n 1}, \ldots, b_{n n}$ :

$$
\begin{aligned}
\sum_{j=1}^{n} u_{n j}\left(\tau_{n i}\right) b_{n j}=f\left(\tau_{n i}\right)+\int_{a}^{b} k\left(\tau_{n i}, s\right) g\left(s, \sum_{j=1}^{n} b_{n j} u_{n j}(s)\right) d s, & \\
& i=1, \ldots, n .
\end{aligned}
$$

Usually, this system of nonlinear equations will have to be solved by some kind of iterative method. It is therefore a marked disadvantage of the above collocation method that the $n$ definite integrals in (1.4) need to be evaluated at each step of the iteration. In practice, these integrals will have to be approximated by a numerical integration technique.

In contrast, an advantage of the method proposed below is that integrals of a similar nature (see (1.10)) need to be calculated once only, and result in a closed set of algebraic nonlinear equations for the $n$ unknowns. In favorable cases (see, for example, the problem studied in Section 5) it may even turn out that the required integrals can be evaluated analytically.

Essentially, the new method involves finding a collocation approximation for the function $z$ defined by

$$
z(t):=g(t, y(t)), \quad t \in[a, b] .
$$

On substituting (1.5) into (1.1) we have immediately

$$
y(t)=f(t)+\int_{a}^{b} k(t, s) z(s) d s, \quad t \in[a, b],
$$

and hence it follows from (1.5) that $z$ satisfies the nonlinear integral equation

$$
z(t)=g\left(t, f(t)+\int_{a}^{b} k(t, s) z(s) d s\right), \quad t \in[a, b] .
$$

In the following section we shall show that this equation is equivalent to (1.1), in the sense of having solutions in one-to-one correspondence with the solutions of (1.1).

The collocation approximation to $z$, by analogy with the standard collocation method described above, is of the form

$$
z_{n}(t)=\sum_{j=1}^{n} a_{n j} u_{n j}(t), \quad t \in[a, b],
$$

where the coefficients $a_{n 1}, \ldots, a_{n n}$ are determined by collocating (1.7) at the collocation points $\tau_{n 1}, \ldots, \tau_{n n}$ :

$$
z_{n}\left(\tau_{n i}\right)=g\left(\tau_{n i}, f\left(\tau_{n i}\right)+\int_{a}^{b} k\left(\tau_{n i}, s\right) z_{n}(s) d s\right), \quad i=1, \ldots, n
$$

or

$$
\sum_{j=1}^{n} u_{n j}\left(\tau_{n i}\right) a_{n j}=g\left(\tau_{n i}, f\left(\tau_{n i}\right)+\sum_{j=1}^{n} \int_{a}^{b} k\left(\tau_{n i}, s\right) u_{n j}(s) d s a_{n j}\right)
$$

$$
i=1, \ldots, n,
$$

which is a closed set of $n$ algebraic nonlinear equations for $a_{n 1}, \ldots, a_{n n}$. 
The required approximation to the solution $y$ of (1.1) is obtained, within our present method, by substituting the approximation $z_{n}$ into the right-hand side of (1.6). That is, the approximation to $y$ is $y_{n}$, where

$$
\begin{aligned}
y_{n}(t) & :=f(t)+\int_{a}^{b} k(t, s) z_{n}(s) d s \\
& =f(t)+\sum_{j=1}^{n} \int_{a}^{b} k(t, s) u_{n j}(s) d s a_{n j} .
\end{aligned}
$$

The principal task in this paper is to show that the approximation $y_{n}$ converges under suitable conditions to an exact solution of (1.1), and to analyze the rate of this convergence. This is carried out in Section 4, the preceding two sections being devoted to establishing the equivalence of (1.1) and (1.7), and to the introduction of some useful smoothness assumptions. The final section examines a numerical example in some detail.

It may be remarked here that the passage from $z_{n}$ to $y_{n}$, achieved in (1.11) by the application of an integral operator, will often give a rather smooth approximation $y_{n}$, even if $z_{n}$ is, say, a piecewise-constant approximation. This might be considered another advantage of the proposed method compared with the conventional collocation method. It may be useful to observe that if $g(s, y(s))=y(s)$, whence (1.1) reduces to a linear integral equation, then the approximation $y_{n}$ becomes what is sometimes called a 'product-integration' approximation [1]. The linear case also suggests another possibility: that under suitable circumstances, the final integration in (1.11) may involve significant cancellation, and so may lead to $y_{n}$ converging to $y$ faster than $z_{n}$ converges to $z$. Since this 'superconvergence' is a subtle question even in the linear case [5]-[8], we choose to defer its consideration to a future paper.

2. Operator Equations for $y$ and $z$. In this section we write the integral equations for $y$ and $z$, Eqs. (1.1) and (1.7), in operator form, and establish that their solutions are in one-to-one correspondence and have certain properties in common.

A convenient setting for the analysis of (1.1) is the space $C=C[a, b]$, the Banach space of continuous functions on $[a, b]$ with the uniform norm

$$
\|x\|=\sup _{a \leqslant t \leqslant b}|x(t)|, \quad x \in C .
$$

On the other hand, it is convenient to analyze (1.7) in the space $L_{\infty}=L_{\infty}(a, b)$, the Banach space of essentially bounded functions on $(a, b)$ equipped with the norm

$$
\|w\|=\underset{a<t<b}{\operatorname{ess} \sup }|w(t)|, \quad w \in L_{\infty},
$$

in order to allow the possibility of discontinuous approximations to $z$.

It is also convenient to make the following assumptions on the functions $f, k$, and $g$ in (1.1):

A1. $f \in C$;

A2. the kernel $k$ satisfies

$$
\sup _{a \leqslant t \leqslant b} \int_{a}^{b}|k(t, s)| d s<\infty
$$

and

$$
\lim _{t \rightarrow t^{\prime}} \int_{a}^{b}\left|k(t, s)-k\left(t^{\prime}, s\right)\right| d s=0, \quad t^{\prime} \in[a, b]
$$


A3. the function $g(t, v)$ is defined and continuous on $[a, b] \times \mathbf{R}$.

Note that under assumption A2 it follows from the Arzelà-Ascoli theorem $[9, \mathrm{p}$. 27] that the linear integral operator $K$, defined by

$$
(K w)(t):=\int_{a}^{b} k(t, s) w(s) d s, \quad t \in[a, b],
$$

is a compact operator from $L_{\infty}$ to $C$, and hence also from $C$ to $C$. Being both compact and linear, it is necessarily (see [9, p. 244]) completely continuous (c.c.).

We also define a nonlinear operator $T$, differing from $K$ merely by an inhomogeneous term:

$$
T(w)(t):=f(t)+(K w)(t), \quad t \in[a, b], w \in L_{\infty} .
$$

Clearly $T$, like $K$, is a c.c. operator from $L_{\infty}$ to $C$.

Finally, we define a 'substitution' operator $G$ by

$$
G(x)(t):=g(t, x(t)), \quad t \in[a, b], x \in C .
$$

The substitution operator is a continuous, bounded operator on $C[13$, p. 81], but it is not c.c. except in the uninteresting case in which the function $g(t, v)$ is independent of $v$.

With the above notation, the integral equations (1.1) and (1.7) may be written as

$$
y=T G(y), \quad y \in C,
$$

and

$$
z=G T(z), \quad z \in L_{\infty} .
$$

Since $T$ is c.c. from $L_{\infty}$ to $C$, and $G$ is continuous and bounded on $C$, it follows [13, p. 74] that $T G$ is c.c. on $C$, and $G T$ is c.c. on $L_{\infty}$.

The following lemma forms the basis of our present method. It establishes the one-to-one correspondence between the solutions of (2.3) and (2.4), a result which has been pointed out by [13, p. 143].

Lemma 1. The sets $\Theta_{T G}:=\{y \in C: T G(y)=y\}$ and $\Theta_{G T}:=\left\{z \in L_{\infty}: G T(z)=\right.$ $z\}$ are in one-to-one correspondence. Specifically, $G$ is a one-to-one operator from $\Theta_{T G}$ onto $\Theta_{G T}$, with inverse $T$.

In the present work we are interested only in solutions $y^{*}$ of (2.3) that are geometrically isolated [10] - that is to say, there exists some ball $\left\{y \in C:\left\|y-y^{*}\right\|\right.$ $\leqslant \alpha\}$, with $\alpha>0$, that contains no solution of (2.3) other than $y^{*}$.

LEMMA 2. If $y^{*}$ is a geometrically isolated solution of (2.3), then $z^{*}:=G\left(y^{*}\right)$ is a geometrically isolated solution of (2.4). Conversely, if $z^{*}$ is a geometrically isolated solution of (2.4), then $y^{*}:=T\left(z^{*}\right)$ is a geometrically isolated solution of (2.3).

Proof. The result follows easily from the continuity of $T$ and $G$.

We shall subsequently make some use of a topological approach to the study of nonlinear equations [11], [13]. In this approach considerable importance attaches to the index of a geometrically isolated solution of an equation such as (2.3). By definition, the index of the geometrically isolated solution $y^{*}$ is the common value of the rotation of the vector field $I-T G$ ( $I$ being the identity) over all sufficiently small spheres centered at $y^{*}$. (For a discussion of rotation, see [13]; in particular, see the properties listed on p. 100.) 
LEMMA 3. Let $y^{*}$ be a geometrically isolated solution of (2.3) and let $z^{*}$ be the corresponding geometrically isolated solution of (2.4). Then $y^{*}$ and $z^{*}$ have the same index.

Proof. This is a special case of Theorem 26.3 of [13].

A geometrically isolated solution of a fixed-point equation is of particular interest if its index is nonzero, since such solutions are generally stable under small perturbations of the problem. We shall exploit this property in the analysis of the approximate method in Section 4. (Geometrically isolated solutions with index zero, on the other hand, are much less favorable for approximate analysis, as may be gathered from a consideration of the solution $x^{*}=0$ of the one-dimensional fixed-point equation $x=x-x^{2}$, which is equivalent to the equation $x^{2}=0$.)

3. Smoothness of $g$. Under mild smoothness assumptions on $g$, the operator $G T$ in (2.4) is Fréchet differentiable. The usefulness of this knowledge becomes apparent in the next section; in brief, it is that it opens the possibility of predicting the rate of convergence of $z_{n}$ to $z^{*}$, and hence of $y_{n}$ to $y^{*}$.

Lemma 4. Suppose A1 to A3 hold. Further, suppose that the partial derivative $g_{v}(t, v):=\partial g(t, v) / \partial v$ exists and is continuous for $a \leqslant t \leqslant b,-\infty<v<\infty$. Then the operator $G$ is continuously Fréchet differentiable on $C$; its Fréchet derivative at $x \in C$ is the multiplicative linear operator $G^{\prime}(x)$ given by

$$
\left[G^{\prime}(x) w\right](t)=g_{v}(t, x(t)) w(t), \quad t \in[a, b], w \in C .
$$

Furthermore, the operator GT is continuously Fréchet differentiable on $L_{\infty}$; its Fréchet derivative at $z \in L_{\infty}$ is the c.c. linear operator $(G T)^{\prime}(z)$ given by

$$
\left[(G T)^{\prime}(z) w\right](t)=g_{v}(t, f(t)+(K z)(t))(K w)(t), \quad t \in[a, b], w \in L_{\infty} .
$$

Proof. The first result is well known [13, p. 81] and the second easily proved using results in [9, p. 499] and [13, p. 77].

The link between the Fréchet derivative of $G T$ and the topological considerations at the end of the preceding section is provided by the following result, which is a special case of a theorem of Leray and Schauder [13, p. 108].

Proposition 1. Assume A1 to A3 hold, and let $g_{v}(t, v)$ exist and be continuous for $a \leqslant t \leqslant b,-\infty<v<\infty$. Further, let $z^{*}$ be a solution of (2.4), and assume that the linear operator $(G T)^{\prime}\left(z^{*}\right)$ does not have 1 as an eigenvalue. Then the solution $z^{*}$ is geometrically isolated and has index \pm 1 .

In particular, under the conditions stated in the proposition, the solution $z^{*}$ is stable under small perturbations. Following Keller [10], we shall say that the solution $z^{*}$ is an isolated solution of (2.4) if Proposition 1 holds. It then follows that an isolated solution is also geometrically isolated and has an index of \pm 1 .

4. Convergence of the Approximate Method. In this section we apply the theory [12], [18], [19] of the approximate solution of nonlinear operator equations by projection methods, to prove the convergence of $z_{n}$ to $z^{*}$, and hence the convergence of $y_{n}$ to $y^{*}$. (For other applications of this theory, see [14], [17], [20].) To this end we first place the collocation procedure for approximating $z^{*}$ within a projection method framework [1], [12], [19] by introducing an interpolatory projection operator $P_{n}$. 
Let the functions $u_{n 1}, \ldots, u_{n n}$ be piecewise continuous on $[a, b]$ (this, of course, includes the continuous case) and such that each is right-continuous at point $a$, and left-continuous at every discontinuity as well as at point $b$. We assume, for simplicity, that

$$
u_{n j}\left(\tau_{n i}\right)=\delta_{i j}, \quad 1 \leqslant i, j \leqslant n,
$$

where $\delta_{i j}$ is the Kronecker delta, since this may always be achieved by an appropriate basis transformation within the subspace $U_{n}:=\operatorname{span}\left\{u_{n 1}, \ldots, u_{n n}\right\} \subset L_{\infty}$.

Let $P_{n}$ be the interpolatory projection operator from $C+U_{n}$ to $U_{n}$, defined, for $w \in C+U_{n}$, by

$$
\left(P_{n} w\right)(t):=\sum_{j=1}^{n} w\left(\tau_{n j}\right) u_{n j}(t), \quad t \in[a, b] .
$$

It may be easily shown that $P_{n}$ is a bounded linear operator on $C+U_{n}$ with image $U_{n}$, that its norm is

$$
\left\|P_{n}\right\|=\sup _{a \leqslant t \leqslant b} \sum_{j=1}^{n}\left|u_{n j}(t)\right|,
$$

and that it has the idempotent property $P_{n}^{2}=P_{n}$.

We shall assume that the subspace $U_{n}$ and the collocation points $\left\{\tau_{n i}\right\}_{i=1}^{n}$ are such that

$$
\lim _{n \rightarrow \infty}\left\|w-P_{n} w\right\|=0 \quad \text { for all } w \in C .
$$

It then follows from the Banach-Steinhaus (or uniform boundedness) theorem [9, p. 203] that $P_{n}$ is uniformly bounded as an operator from $C$ to $U_{n} \subset L_{\infty}$. Hence (since $\left\|P_{n}\right\|_{C \rightarrow U_{n}}$ is equal to the right-hand side of (4.3))

$$
\left\|P_{n}\right\| \leqslant c_{1}
$$

where $c_{1}>0$ is independent of $n$.

Using the operator $P_{n}$, we may write the collocation equations (1.8) and (1.9) as the single equation

$$
z_{n}=P_{n} G T\left(z_{n}\right), \quad z_{n} \in U_{n} \subset L_{\infty} .
$$

From Lemmas 1 to 4 and a direct application of Theorem 19.7 of [12] we now obtain

THEOREM 1. Let $y^{*} \in C$ be a geometrically isolated solution of (2.3), and let $z^{*}$ be the corresponding solution of (2.4). Suppose A1 to A3 hold, and that the interpolatory operator $P_{n}$ satisfies (4.4).

(i) If $y^{*}$ has a nonzero index, then there exists an $n_{0}$ such that for $n \geqslant n_{0},(4.6)$ has a solution $z_{n} \in U_{n}$ satisfying

$$
\left\|z^{*}-z_{n}\right\| \rightarrow 0 \text { as } n \rightarrow \infty \text {. }
$$

(ii) Suppose that the partial derivative $g_{v}(t, v)$ exists and is continuous for $a \leqslant t \leqslant b$, $-\infty<v<\infty$, and that 1 is not an eigenvalue of the linear operator $(G T)^{\prime}\left(z^{*}\right)$. Then there exists a neighborhood of $z^{*}$ and an $n_{1}$ such that for $n \geqslant n_{1}$ a solution $z_{n}$ of (4.6) is unique in that neighborhood, and

$$
c_{2}\left\|z^{*}-P_{n} z^{*}\right\| \leqslant\left\|z^{*}-z_{n}\right\| \leqslant c_{3}\left\|z^{*}-P_{n} z^{*}\right\|,
$$

where $c_{2}, c_{3}>0$ are independent of $n$. 
COROLlaRY 1. Under the conditions in Theorem 1(ii) there exists a constant $c_{4}>0$ such that

$$
\left\|z^{*}-z_{n}\right\| \leqslant c_{4} \inf _{\phi \in U_{n}}\left\|z^{*}-\phi\right\|
$$

Proof. For any $\phi \in U_{n}$,

$$
\left\|z^{*}-P_{n} z^{*}\right\|=\left\|\left(I-P_{n}\right)\left(z^{*}-\phi\right)\right\| \leqslant\left(1+\left\|P_{n}\right\|\right)\left\|z^{*}-\phi\right\| .
$$

The result now follows from the uniform boundedness of the operator $P_{n}$.

Thus we see that $z_{n}$ converges optimally to $z^{*}$, in the sense that it converges to $z^{*}$ with the same asymptotic order as the best approximation to $z^{*}$ from $U_{n}$.

A convergence result for $y_{n}$ is now easily obtained.

THEOREM 2. Let $y^{*} \in C$ be a geometrically isolated solution of (2.3), and let $z^{*}$ be the corresponding solution of (2.4). Suppose A1 to A3 hold, and that the interpolatory operator $P_{n}$ satisfies (4.4).

(i) If $y^{*}$ has a nonzero index, then with $z_{n}$ as in Theorem 1(i), and $n \geqslant n_{0}$, (1.11) defines an approximation $y_{n} \in C$ satisfying

$$
\left\|y^{*}-y_{n}\right\| \rightarrow 0 \text { as } n \rightarrow \infty \text {. }
$$

(ii) Suppose that the partial derivative $g_{v}(t, v)$ exists and is continuous for $a \leqslant t \leqslant b$, $-\infty<v<\infty$, and that 1 is not an eigenvalue of the linear operator $(G T)^{\prime}\left(z^{*}\right)$. Then for $n \geqslant n_{1}$ the approximation $y_{n}$ given by (1.11), with $z_{n}$ as in Theorem 1(ii), satisfies

$$
\left\|y^{*}-y_{n}\right\| \leqslant c_{5} \inf _{\phi \in U_{n}}\left\|z^{*}-\phi\right\|
$$

where $c_{5}>0$ is independent of $n$.

Proof. (i) Since $T$ maps $L_{\infty}$ to $C$, it follows immediately from the definition $y_{n}:=T\left(z_{n}\right)$ that $y_{n} \in C$. Now from Lemma 1 ,

$$
y^{*}=T\left(z^{*}\right)=f+K z^{*},
$$

and hence

$$
\left\|y^{*}-y_{n}\right\|=\left\|K\left(z^{*}-z_{n}\right)\right\| \leqslant\|K\|\left\|z^{*}-z_{n}\right\| \rightarrow 0 \quad \text { as } n \rightarrow \infty,
$$

where

$$
\|K\|=\sup _{a \leqslant t \leqslant b} \int_{a}^{b}|k(t, s)| d s<\infty
$$

(ii) This follows similarly, with the aid of Corollary 1.

Thus the rate of convergence of $y_{n}$ to $y^{*}$ is, at the very least, equal to the rate of convergence of the best approximation to $z^{*}$ from $U_{n}$.

5. A Numerical Example. In this section the proposed method is used to solve an integral equation reformulation of the nonlinear two-point boundary value problem

$$
y^{\prime \prime}(t)-\exp (y(t))=0, \quad t \in(0,1) ; \quad y(0)=y(1)=0,
$$

which evidently is of some interest in magnetohydrodynamics [3, p. 41]. This problem has the unique solution

$$
y^{*}(t)=-\ln (2)+2 \ln \left(c / \cos \left(c\left(t-\frac{1}{2}\right) / 2\right)\right)
$$


where $c$ is the only solution of $c / \cos (c / 4)=\sqrt{2}$, and may be reformulated as the integral equation

$$
y(t)=\int_{0}^{1} k(t, s) \exp (y(s)) d s, \quad t \in[0,1]
$$

where the kernel

$$
k(t, s)= \begin{cases}-s(1-t), & s \leqslant t \\ -t(1-s), & s>t\end{cases}
$$

is the Green's function for the homogeneous problem

$$
y^{\prime \prime}(t)=0, \quad t \in(0,1) ; \quad y(0)=y(1)=0 .
$$

Equation (5.1) was solved by the new method, with the collocation points chosen to be $\tau_{n j}=(j-1) /(n-1), j=1, \ldots, n$, and the basis functions $u_{n 1}, \ldots, u_{n n}$ defined as follows:

$$
\begin{gathered}
u_{n 1}(t)= \begin{cases}\left(t-\tau_{n 2}\right) /\left(\tau_{n 1}-\tau_{n 2}\right), & t \in\left[\tau_{n 1}, \tau_{n 2}\right], \\
0, & \text { otherwise; }\end{cases} \\
u_{n j}(t)=\left\{\begin{array}{ll}
\left(t-\tau_{n, j-1}\right) /\left(\tau_{n j}-\tau_{n, j-1}\right), & t \in\left(\tau_{n, j-1}, \tau_{n j}\right] \\
\left(t-\tau_{n, j+1}\right) /\left(\tau_{n j}-\tau_{n, j+1}\right), & t \in\left(\tau_{n j}, \tau_{n, j+1}\right] \\
0, & \text { otherwise }
\end{array}\right\}, \quad j=2, \ldots, n-1 ; \\
u_{n n}(t)=u_{n 1}(1-t) .
\end{gathered}
$$

That is, the solution $z^{*}(t)=\exp \left(y^{*}(t)\right)$ of the integral equation

$$
z(t)=\exp \left(\int_{0}^{1} k(t, s) z(s) d s\right), \quad t \in[0,1]
$$

was approximated by the process of continuous piecewise-linear collocation [8].

Note that for the above choice of the basis functions and collocation points, $P_{n}$ is a uniformly bounded operator on $C[0,1]$ and (4.4) holds [21]. Notice that in this case (4.1) also holds, and so the nonlinear system corresponding to (1.10) is simply

$$
a_{n i}=\exp \left(\sum_{j=1}^{n} \int_{0}^{1} k\left(\tau_{n i}, s\right) u_{n j}(s) d s a_{n j}\right), \quad i=1, \ldots, n .
$$

The integrals in (5.2) (and later those in (1.11)) were calculated exactly, and the system (5.2) was then solved for the unknowns $a_{n 1}, \ldots, a_{n n}$ by Moré and Cosnard's implementation [16] of a modification [15] of Brent's method [4]. All computations were carried out in double precision on a Vax 11/750 computer.

It is well known that approximation by piecewise-linear functions yields, at best, $O\left(h^{2}\right)$ convergence, where $h$ is the maximum distance between the breakpoints of the pieces. Thus from Theorems 1 and 2 we expect

$$
\left\|z^{*}-z_{n}\right\|=O\left(h^{\xi_{1}}\right), \quad\left\|y^{*}-y_{n}\right\|=O\left(h^{\xi_{2}}\right),
$$

with $\xi_{1}=\xi_{2}=2$. This is confirmed by the results in Table I.

The maximum errors listed in Table I were estimated by taking the largest of the computed errors at $t=(i-1) / 256, i=1, \ldots, 129$. (Here we have used the fact that (5.1) is symmetric about $t=\frac{1}{2}$.) Estimates of $\xi_{1}$ were obtained by using the formula

$$
\xi_{1} \simeq \ln \left(\left\|z^{*}-z_{n}\right\| /\left\|z^{*}-z_{2 n-1}\right\|\right) / \ln (2) .
$$

The estimates of $\xi_{2}$ were calculated similarly. 
TABLE I

\begin{tabular}{rcccc}
\hline$n$ & $\left\|z^{*}-z_{n}\right\|$ & Estimate of $\xi_{1}$ & $\left\|y^{*}-y_{n}\right\|$ & Estimate of $\xi_{2}$ \\
\hline 5 & $7.81 E-3$ & 1.87 & $5.19 E-4$ & 2.02 \\
9 & $2.13 E-3$ & 1.93 & $1.28 E-4$ & 2.00 \\
17 & $5.61 E-4$ & 1.96 & $3.20 E-5$ & 2.00 \\
33 & $1.44 E-4$ & 1.98 & $7.99 E-6$ & 2.00 \\
65 & $3.66 E-5$ & & $2.00 E-6$ & \\
\hline
\end{tabular}

Acknowledgments. The authors are indebted to Dr. J. D. Gray and Dr. A. Spence for comments on an earlier draft.

School of Mathematics

University of New South Wales

Sydney, N.S.W. 2033, Australia

1. K. E. AtKinson, A Survey of Numerical Methods for the Solution of Fredholm Integral Equations of the Second Kind, SIAM, Philadelphia, Pa., 1976.

2. C. T. H. BAKER, The Numerical Treatment of Integral Equations, Clarendon Press, Oxford, 1977.

3. R. E. Bellman \& R. E. Kalaba, Quasilinearization and Nonlinear Boundary-Value Problems, Elsevier, New York, 1965.

4. R. P. BRENT, "Some efficient algorithms for solving systems of nonlinear equations," SIAM J. Numer. Anal., v. 10, 1973, pp. 327-344.

5. F. Chatelin \& R. LebBaR, "The iterated projection solution for Fredholm integral equations of second kind," J. Austral. Math. Soc. Ser. B, v. 22, 1981, pp. 439-451.

6. F. Chatelin \& R. LebBar, "Superconvergence results for the iterated projection method applied to a Fredholm integral equation of the second kind and the corresponding eigenvalue problem," J. Integral Equations, v. 6, 1984, pp. 71-91.

7. I. G. Graham, S. Joe \& I. H. Sloan, "Iterated Galerkin versus iterated collocation for integral equations of the second kind," IMA J. Numer. Anal., v. 5, 1985, pp. 355-369.

8. S. JoE, "Collocation methods using piecewise polynomials for second kind integral equations," $J$. Comput. Appl. Math., v. $12 \& 13,1985$, pp. 391-400.

9. L. V. Kantorovich \& G. P. AKILOV, Functional Analysis, Pergamon Press, Oxford, 1982.

10. H. B. Keller, "Geometrically isolated nonisolated solutions and their approximation," SIAM J. Numer. Anal., v. 18,1981 , pp. 822-838.

11. M. A. Krasnoser'SKII, Topological Methods in the Theory of Nonlinear Integral Equations, Pergamon Press, Oxford, 1964.

12. M. A. Krasnosel'skil, G. M. VainikKo, P. P. ZabreIko, Ya. B. Rutitskil \& V. Ya. Stetsenko, Approximate Solution of Operator Equations, Wolters-Noordhoff, Groningen, 1972.

13. M. A. Krasnosel'skil \& P. P. ZabreǏKo, Geometrical Methods of Nonlinear Analysis, SpringerVerlag, Berlin, 1984.

14. T. R. LuCAS \& G. W. ReDdien, "Some collocation methods for nonlinear boundary value problems," SIAM J. Numer. Anal., v. 9, 1972, pp. 341-356.

15. J. J. MORE \& M. Y. COSNARD, "Numerical solution of nonlinear equations," ACM Trans. Math. Software, v. 5, 1979, pp. 64-85.

16. J. J. MorE \& M. Y. CoSNARD, "ALGORITHM 554: BRENTM, A Fortran subroutine for the numerical solution of systems of nonlinear equations," ACM Trans. Math. Software, v. 6, 1980, pp. 240-251.

17. R. D. Russell \& L. F. Shampine, "A collocation method for boundary value problems," Numer. Math., v. 19, 1972, pp. 1-28.

18. G. VAINIKKo, "The convergence of the collocation method for nonlinear differential equations," U.S.S.R. Comput. Math. and Math. Phys., v. 6, no. 1, 1966, pp. 47-58.

19. G. VAINIKKo, "Galerkin's perturbation method and the general theory of approximate methods for nonlinear equations," U.S.S.R. Comput. Math. and Math. Phys., v. 7, no. 4, 1967, pp. 1-41.

20. G. VAINIKKo, "The connection between mechanical quadrature and finite difference methods," U.S.S.R. Comput. Math. and Math. Phys., v. 9, no. 2, 1969, pp. 1-16.

21. G. VAINIKKo \& P. UBA, "A piecewise polynomial approximation to the solution of an integral equation with weakly singular kernel," J. Austral. Math. Soc. Ser. B, v. 22, 1981, pp. 431-438. 